\title{
Restoration of optical objects using regularization
}

\author{
M. Bertero \\ Istituto di Scienze Fisiche dell' Università and Istituto Nazionale di Fisica Nucleare, Genova, Italy \\ C. De Mol \\ Département de Mathématique, Université Libre de Bruxelles, Bruxelles, Belgium \\ G. A. Viano \\ Istituto di Scienze Fisiche dell' Università and Istituto Nazionale di Fisica Nucleare, Genova, Italy \\ Received November 16, 1977. Revised manuscript received March 30, 1978
}

\begin{abstract}
Using the regularization theory for improperly posed problems, we discuss object restoration beyond the diffraction limit in the presence of noise. Only the case of one-dimensional coherent objects is considered. We focus attention on the estimation of the error on the restored objects, and we show that, in most realistic cases, it is at best proportional to an inverse power of $|\ln \epsilon|$, where $\epsilon$ is the error on the data (logarithmic continuity). Finally we suggest the extension of this result to other inverse problems.
\end{abstract}

Let us consider an ideal, diffraction-limited, spaceinvariant imaging system and a one-dimensional, coherent object $f$, identically zero outside the interval $(-1,1)$. Its noiseless image is given by $A f$, where $A$ is the linear integral operator,

$$
(A f)(x)=\int_{-1}^{1} \frac{\sin \left[c\left(x-x^{\prime}\right)\right]}{\pi\left(x-x^{\prime}\right)} f\left(x^{\prime}\right) \mathrm{d} x^{\prime} .
$$

The quantity $d=\pi / c$ is the Rayleigh-resolution distance. To restore the object, one has to invert the operator $A$. As is known, this problem is improperly posed. When the image is known only approximately, we have numerical instability. Hence, in order to control error propagation, one has to introduce $a$ priori constraints (as far as possible of physical origin) that restrict the class of admitted solutions. This is the concept of regularization of Tikhonov. Its importance for the practical solution of linear inverse problems has been emphasized by many authors. ${ }^{1-5}$ Various regularization methods have been proposed. ${ }^{6-10}$ They are essentially equivalent, but most of them overlook the problem of obtaining precise estimates on error propagation: usually the stability of the regularized solution is tested only numerically. ${ }^{1-3,7}$ Our aim is to focus on error valuation, and therefore we shall use regularization in a formulation of Miller, ${ }^{9}$ which is particularly suited for deriving stability estimates.

In order to formulate our problem more precisely, we have to introduce suitable functional spaces for the solutions and for the data. Now, if the object $f$ is taken to have finite energy, then it belongs to $L^{2}(-1,1)$ (hereafter abbreviated as $L^{2}$ ). We denote by $(f, g)$ the scalar product of two functions of $L^{2}$, i.e.,

$$
(f, g)=\int_{-1}^{1} f(x) g^{*}(x) \mathrm{d} x
$$

where $g^{*}$ is the complex conjugate of $g$, and by $\|f\|=$ $(f, f)^{1 / 2}$ the $L^{2}$ norm of $f$. Moreover, we suppose that the optical image is observed in the interval $(-1,1)$ so that we can again take $L^{2}$ as data space.

If $g$ denotes a noisy image corresponding to the object $f$, then we assume that $g=A f+n$, where $n$ is an $L^{2}$ function describing any kind of additive noise and errors. This particular model is unrealistic; nonetheless it is the only one found usable to date. ${ }^{11}$ Obviously $n$ is not known. However, we assume an upper bound for its $L^{2}$ norm, i.e., $\|n\| \leq \epsilon$. Besides, according to Miller, ${ }^{9}$ we assume that the additional information on the object $f$ (which is required in order to get numerical stability) may be expressed by means of a constraint operator $B$ as follows: $\|B f\| \leq E$, where $E$ is a known constant. Thus we have:

$$
\begin{gathered}
\|A f-g\| \leq \epsilon, \\
\|B f\| \leq E .
\end{gathered}
$$

The simplest choice for $B$ is $B=1$ (the identity operator). $., 2,4,8$ Then, in object restoration, condition (3b) means that.we assume an upper bound for the total energy of the object. Another usual choice is to let $B$ be a differential operator. ${ }^{3,6-8}$ Then condition (3b) gives a bound on some derivatives of $f$ and hence provides a smoothness requirement on the object. At this stage, however, we do not yet specify $B$.

Now let us denote by $F$ the set of all the functions satisfying conditions (3a) and (3b). We can take as an approximation of the unknown object $f$ any function $\tilde{f}$ belonging to $F$. To determine the accuracy of this approximation, we must define a distance between two functions of $F$. A natural choice (which is not the only possible one; see below) is to take the distance induced by the $L^{2}$ norm so that the error is $\|\tilde{f}-f\|$. Since $f$ is not known, we can say only that the error is at most the 
maximum distance between any two functions of $F$. Moreover, it can be shown ${ }^{9}$ that this maximum distance is bounded by a quantity depending only on the numbers $\epsilon$ and $E$, i.e., $\|\tilde{f}-f\| \leq 2 M(\epsilon, E)$, where $M(\epsilon, E)$ is the maximum norm of the functions satisfying conditions (3a) and (3b) with $g=0$ [i.e., $M(\epsilon, E)=\sup _{f}\|f\|$, when $\|A f\| \leq \epsilon,\|B f\| \leq E]$. This is the best upper bound we can prescribe for the error, and hence $M(\epsilon, E)$ is called the best possible stability estimate. ${ }^{9}$ When, for fixed $E, M(\epsilon, E)$ tends to zero as $\epsilon$ tends to zero, any approximation $\tilde{f}$ converges to the true object $f$. Then we say that we have obtained continuous dependence of the solution on the data.

We sketch here Miller's regularization method, ${ }^{9}$ and, for simplicity, we assume that we know both constants $\epsilon$ and $E$ (however, this requirement is not indispensable; see Miller's paper ${ }^{9}$ ). We remark first that any function $\tilde{f}$ satisfying conditions (3a) and (3b) also satisfies the following one:

$$
\Phi(\tilde{f})=\|A \tilde{f}-g\|^{2}+(\epsilon / E)^{2}\|B \tilde{f}\|^{2} \leq 2 \epsilon^{2} .
$$

Any $L^{2}$ function satisfying Eq. (4) also satisfies conditions (3a) and (3b), except for a factor of at most $\sqrt{2}$. When $B$ has a bounded inverse, there exists one and only one function $f_{1}$ minimizing $\Phi(\operatorname{Ref} .9)$ :

$$
f_{1}=\left[A^{*} A+(\epsilon / E)^{2} B^{*} B\right]^{-1} A^{*} g,
$$

where $A^{*}$ and $B^{*}$ are the adjoints of $A$ and $B$. Then, since $f_{1}$ satisfies Eq. (4), we can take $f_{1}$ as an estimate of the unknown object $f$. The function $f_{1}$ is similar to Tikhonov's solution, $1,6,8,10$ if we note that the regularization parameter is given here by $(\epsilon / E)^{2}$, while it remains free in Tikhonov's method. Besides, Eq. (5) is similar to a Wiener filter.

Now, to be able to compute the stability estimates explicitly, we have to assume further that the operators $A$ and $B$ commute.

Let us recall that operator $A[E q$. (1)] has a complete orthonormal set of eigenfunctions in $L^{2}: u_{k}(x)=$ $\lambda_{k}{ }^{-1 / 2} \psi_{k}(x)(k=0,1,2, \ldots)$, where $\psi_{k}(x)$ are the linear prolate spheroidal wavefunctions ${ }^{12}$ and $\lambda_{k}$ are the corresponding eigenvalues. $\psi_{k}$ have their $L^{2}$ norm equal to $\lambda_{k}{ }^{1 / 2}$ and satisfy the differential equation, ${ }^{12,13}$

$$
-\left[\left(1-x^{2}\right) \psi_{k}\right]^{\prime}+c^{2} x^{2} \psi_{k}=\chi_{k} \psi_{k},
$$

where $\chi_{k}=k(k+1)+1 / 2\left(c^{2}\right)+O\left(k^{-2}\right)$ for $k \rightarrow \infty$. When $B$ commutes with $A$, we may expand it as follows:

$$
(B f)(x)=\sum_{k=0}^{+\infty} \beta_{k} f_{k} u_{k}(x),
$$

where $f_{k}=\left(f, u_{k}\right)$ are the Fourier coefficients of $f$ with respect to the functions $u_{k}$. The operator $B$ has a bounded inverse if and only if $\beta_{k} \neq 0$ for any $k$, and $(\sup / k)\left(\beta_{k}-1\right)<+\infty$. Then Eq. (5) becomes:

$$
f_{1}(x)=\sum_{k=0}^{+\infty} \frac{\lambda_{k}}{\lambda_{k}{ }^{2}+(\epsilon / E)^{2} \beta_{k}^{2}} g_{k} u_{k}(x),
$$

where $g_{k}=\left(g, u_{k}\right)$. Equation (8) coincides formally with minimum mean-squared-error linear estimates. 1,4,14,15 Another simple estimate, $f_{2}$, can be obtained as follows. Let us recall that the $\lambda_{k}$ 's form a decreasing sequence converging to zero and denote by $k_{0}$ the greatest integer such that $\lambda_{k} \geq \epsilon \beta_{k} / E$. Then we write

$$
f_{2}(x)=\sum_{k=0}^{k_{0}} \frac{g_{k}}{\lambda_{k}} u_{k}(x) .
$$

We recover here with a prescribed cutoff (which, for $B$ $=1$, is essentially the stability criterion of Cabayan $e t$ $\left.a l .{ }^{2}\right)$ the well-known truncation method for eliminating the noise amplification due to eigenvalues $\lambda_{k}$ very close to zero. ${ }^{11,15}$ Both estimates $f_{1}$ and $f_{2}$ can be shown to satisfy the inequality $\left\|_{i}-f\right\| \leq \sqrt{2} M(\epsilon, E),(i=1,2)$, and therefore they converge to the true object $f$ when $M(\epsilon, E)$ tends to zero.

We shall illustrate now some results that we have obtained about stability estimates. Further details and mathematical proofs will be published elsewhere. ${ }^{16}$

It can be proved that the stability estimate $M(\epsilon, E)$, as defined above, tends to zero when $\epsilon$ tends to zero for fixed $E$, if and only if $\lim _{k \rightarrow \infty} \beta_{k}=\infty$. Obviously this condition is not satisfied by $B=1$, which is the constraint operator taken by most authors (see above). However, this fact does not prevent any kind of stability of the solution. Indeed, it is possible to prove ${ }^{9,16}$ that, when $B=1$, we have stability if we measure the closeness of the functions $\tilde{f}$ and $f$ (see above) by means of weighted averages of their difference. For instance, for any $x_{0} \epsilon(-1,1)$ and for fixed $\delta$, all the quantities

$$
\left|\int_{-1}^{1}[\tilde{f}(x)-f(x)] \exp \left[-\left(\frac{x-x_{0}}{\delta}\right)^{2}\right] \mathrm{d} x\right|
$$

tend to zero when $\epsilon$ tends to zero. In other words, we can restore any local average of $f$ over some resolving length $\delta$.

Our present purpose is to discuss stability with respect to the norm of $L^{2}$, which implies in particular the kind of stability just described. Besides, as emphasized by John, ${ }^{17}$ it is important for practical purposes to determine how fast $M(\epsilon, E)$ tends to zero when $\epsilon$ tends to zero.

\section{Hölder Continuity}

Let us take first $B=A^{-1}$, i.e., $\beta_{k}=\lambda_{k}^{-1}$, so that condition (3b) becomes $\left\|A^{-1} f\right\| \leq E$. Schwarz's inequality implies that $\|f\|^{2}=(f, f)=\left(A f, A^{-1} f\right) \leq\|A f\| \times\left\|A^{-1} f\right\|$. Thus we have $M(\epsilon, E) \leq(\epsilon E)^{1 / 2}$ [the equal sign holds when $(\epsilon / E)^{1 / 2}$ is equal to one of the eigenvalues of the operator $A]$. When the stability estimate tends to zero as $\epsilon^{\alpha}(0<\alpha \leq 1)$, one says that the solution of the problem depends Hölder-continuously on the data. This kind of continuity is fairly satisfactory, since it means that only a fixed percentage of significant digits need be lost in determining $f$ from $g$.

Unfortunately the condition $\left\|A^{-1} f\right\| \leq E$ is very restrictive; indeed, it implies that $f$ is the restriction [to the interval $(-1,1)$ ] of an analytic function and hence is already smooth before imaging. The same conclusion follows when one recalls the step-function behavior of the eigenvalues $\lambda_{k}$ : they remain approximately equal to 1 for $k<2 c / \pi$ and fall off to zero very rapidly for $k>$ $2 c / \pi$. Hence condition $\left\|A^{-1} f\right\| \leq E$ defines a class of objects whose Fourier components $f_{k}$ are significant only for $k<2 c / \pi$. Since the image has significant components only for $k<2 c / \pi$, we understand easily why the restored continuity is quite good. 


\section{Logarithmic Continuity}

Next we consider the case for which the $\beta_{k}$ 's grow like a power of $k$. In order to clarify what this constraint means, let us remark that, if we take $\beta_{k}=\chi_{k}{ }^{1 / 2} \sim k, k$ $\rightarrow \infty\left[\chi_{k}\right.$ being the eigenvalues of differential Eq. (6)], we easily deduce from Eq. (6) that

$$
\begin{aligned}
\|B f\|^{2}=\int_{-1}^{1}\left(1-x^{2}\right)\left|f^{\prime}(x)\right|^{2} \mathrm{~d} x & \\
& +c^{2} \int_{-1}^{1} x^{2}|f(x)|^{2} \mathrm{~d} x .
\end{aligned}
$$

Therefore we essentially get a bound on the first derivative of the object $f$. Similarly, taking $\beta_{k} \sim k^{N}$, we get bounds on the derivatives of $f$ up to the order $N$. For such a constraint operator $B$, we can prove that ${ }^{16}$

$$
M(\epsilon, E) \sim(\text { constant }) \times E|\ln (\epsilon / E)|^{-(N-\eta)}
$$

(where $\eta$ may be made as small as desired), provided that the ratio $\epsilon / E$ be sufficiently small (the result is asymptotic in the limit $\epsilon \rightarrow 0$ ).

One can convince oneself of the validity of formula (12) by the following argument. As indicated by the truncated solution (9), we can compute only those components of the object whose index $k$ is such that $\lambda_{k}$ $\geq \epsilon \beta_{k} / E \sim \epsilon k^{N} / E$ (compare also the results of Twomey ${ }^{8}$ and of Cabayan et al. ${ }^{2}$ ). The number $k_{0}$ of these components is roughly of the order of $|\ln \epsilon|$, since the eigenvalues $\lambda_{k}$ decrease exponentially fast. ${ }^{18}$ Now, when we compute only $k_{0}$ components of an object $f$ satisfying condition ( $3 \mathrm{~b})$, we make an error that is roughly the maximum value (allowed by the constraint) of the first neglected term, i.e., $\left(E / \beta_{k_{0}+1}\right) \sim k_{0}{ }^{-N} \sim|\ln \epsilon|^{-N}$.

The kind of continuity implied by formula (12) is called logarithmic continuity and is, in practice, very poor. For example, with $N=1, E$ fixed, and $\epsilon / E$ initially equal to $10^{-1}$, an increase in solution accuracy by factor of $2,3,4 \ldots$ would require an increase in the data accuracy to $\epsilon / E=10^{-2}, 10^{-3}, 10^{-4} \ldots$, etc. Of course, since formula (12) is only asymptotic, this remark is true when we want to reconstruct those components $f_{k}$ whose index is much greater than $2 c / \pi$, i.e., when we want to go far beyond the Rayleigh limit. In other words, this result does not contradict the conclusion of previous analysis that, "if the object is very poorly resolved by the optical system at the start, a significant improvement in resolution can be accomplished if reasonably high signal-to-noise ratios are available before processing". ${ }^{11}$ This means that the addition of a few resolvable elements is possible. However, if we want to resolve more and more components $f_{k}$, then the required signal-to-noise ratios are unrealistically high.

The result we got here derives essentially from the rapid exponential fall of the eigenvalues $\lambda_{k}$ of the operator $A$. An asymptotic behavior of the same kind [more precisely, $\lambda_{k} \sim \exp (-D k \ln k)$, where $D$ is some constant] holds true for every integral operator whose kernel is an entire analytic function of finite order. ${ }^{19}$ So we may argue that we get at best logarithmic continuity whenever, for inverting such an integral operator, we impose $a$ priori bounds on a finite number of derivatives of the solutions. For instance, analytic kernels are involved in the problem of near-field reconstruction ${ }^{2}$ and in the Bojarski-Lewis inverse-scattering method when one has information only over a finite frequency band (bandpass kernels). ${ }^{4}$

We are deeply indebted to a referee for helpful remarks and valuable bibliographical information. We also thank A. Consortini, L. Ronchi, and G. Toraldo di Francia for many interesting discussions. C. DeMol is an Aspirant du Fonds National Belge de la Recherche Scientifique.

\section{References}

1. G. A. Deschamps and H. S. Cabayan, "Antenna synthesis and solution of inverse problems by regularization methods," IEEE Trans. Antennas Propag. AP-20, 268-274 (1972).

2. H. S. Cabayan, R. C. Murphy, and T. J. F. Pavlásek, "Numerical stability and near-field reconstruction," IEEE Trans. Antennas Propag. AP-21, 346-351 (1973).

3. R. Barakat and E. Blackman, "Application of the Tikhonov regularization algorithm to object restoration," Opt. Commun. 9, 252-256 (1973).

4. W. L. Perry, "On the Bojarski-Lewis inverse scattering method," IEEE Trans. Antennas Propag. AP-22, 826-829 (1974).

5. G. A. Viano, "On the extrapolation of optical image data," J. Math. Phys. 17, 1160-1165 (1976).

6. A. Tikhonov, "Solution of incorrectly formulated problems and the regularization method," Sov. Math. 4, 1035-1038 (1963); "Regularization of incorrectly posed problems," Sov. Math. 4, 1624-1627 (1963).

7. B. L. Phillips, "A technique for the numerical solution of certain integral equations of the first kind," J. Assoc. Comput. Mach. 9, 84-97 (1962).

8. S. Twomey, "The application of numerical filtering to the solution of integral equations encountered in indirect sensing measurements," J. Franklin Inst. 279, 95-109 (1965).

9. K. Miller, "Least squares method for ill-posed problems with a prescribed bound," SIAM J. Math. Anal. 1, 52-74 (1970).

10. M. Z. Nashed and G. Wahba, "Generalized inverses in reproducing kernel spaces: an approach to regularization of linear operator equations," SIAM J. Math. Anal. 5, 974-987 (1974).

11. J. W. Goodman, "Synthetic-aperture optics," in Progress in Optics, E. Wolf, ed. (North-Holland, Amsterdam, 1970), Vol. VIII, pp. 1-50, Sec. 6.

12. D. Slepian and H. O. Pollak, "Prolate spheroidal wave functions, Fourier analysis and uncertainty (I)," Bell Syst. Tech. J. 40, 43-63 (1961).

13. J. Meixner and F. W. Schäfke, Mathieusche Funktionen und Sphäroidfunktionen (Springer, Berlin, 1954).

14. G. J. Buck and J. J. Gustincic, "Resolution limitations of a finite aperture," IEEE Trans. Antennas Propag. AP-15, 376-381 (1967).

15. C. K. Rushforth and R. W. Harris, "Restoration, resolution, and noise," J. Opt. Soc. Am. 58, 539-545 (1968).

16. M. Bertero, C. DeMol, and G. A. Viano, "On the problems of object restoration and image extrapolation in optics" (submitted to J. Math. Phys.).

17. F. John, "Continuous dependence on data for solutions of partial differential equations with prescribed bound," Commun. Pure Appl. Math. 13, 551-585 (1960).

18. H. J. Landau, "The eigenvalue behavior of certain convolution equations," Trans. Am. Math. Soc. 115, 242-256 (1965).

19. E. Hille and J. D. Tamarkin, "On the characteristic values of linear integral equations," Acta Math. 57, 1-76 (1931). 